\title{
Effects of Nebulizer Position, Gas Flow, and CPAP on Aerosol Bronchodilator Delivery: An In Vitro Study
}

\author{
Lorenzo Ball MD, Yuda Sutherasan MD, Valentina Caratto PhD, Elisa Sanguineti PhD, \\ Maria Marsili MD, Pasquale Raimondo MD, Maurizio Ferretti, \\ Robert M Kacmarek PhD RRT FAARC, and Paolo Pelosi MD
}

\begin{abstract}
BACKGROUND: The aim of this study was to investigate the effects of different delivery circuit configurations, nebulizer positions, CPAP levels, and gas flow on the amount of aerosol bronchodilator delivered during simulated spontaneous breathing in an in vitro model. METHODS: A pneumatic lung simulator was connected to 5 different circuits for aerosol delivery, 2 delivering CPAP through a high-flow generator tested at 30, 60, and $90 \mathrm{~L} / \mathrm{min}$ supplementary flow and 5, 10, and $15 \mathrm{~cm} \mathrm{H}_{2} \mathrm{O}$ CPAP and 3 with no CPAP: a T-piece configuration with one extremity closed with a cap, a T-piece configuration without cap and nebulizer positioned proximally, and a T-piece configuration without cap and nebulizer positioned distally. Albuterol was collected with a filter, and the percentage amount delivered was measured by infrared spectrophotometry. RESULTS: Configurations with continuous high-flow CPAP delivered higher percentage amounts of albuterol compared with the configurations without CPAP $(9.1 \pm 6.0 \%$ vs $6.2 \pm 2.8 \%, P=.03)$. Among configurations without CPAP, the best performance was obtained with a T-piece with one extremity closed with a cap. In CPAP configurations, the highest delivery $(13.8 \pm 4.4 \%)$ was obtained with the nebulizer placed proximal to the lung simulator, independent of flow. CPAP at $15 \mathrm{~cm} \mathrm{H}_{2} \mathrm{O}$ resulted in the highest albuterol delivery $(P=.02)$. CONCLUSIONS: Based on our in vitro study, without CPAP, a T-piece with a cap at one extremity maximizes albuterol delivery. During high-flow CPAP, the nebulizer should always be placed proximal to the patient, after the T-piece, using the highest CPAP clinically indicated. Key words: aerosol; bronchodilators; albuterol; continuous positive airway pressure; cpap; nebulizers. [Respir Care 2016;61(3):263-268. @ 2016 Daedalus Enterprises]
\end{abstract}

\section{Introduction}

Nebulized bronchodilators are frequently administered to mechanically ventilated and spontaneously breathing

\footnotetext{
Drs Ball, Sutherasan, Marsili, Raimondo, and Pelosi are affiliated with IRCCS AOU San Martino-IST, Department of Surgical Sciences and Integrated Diagnostics, University of Genoa, Genoa, Italy. Dr Sutherasan is also affiliated with the Division of Pulmonary and Critical Care Medicine, Faculty of Medicine, Ramathibodi Hospital, Mahidol University, Bangkok, Thailand. Dr Raimondo is also affiliated with the Department of Anesthesiology, Resuscitation, and Intensive Care, Università degli Studi di Foggia, Foggia, Italy. Dr Caratto, Dr Sanguineti, and Mr Ferretti are affiliated with the Department of Chemistry and Industrial Chemistry, University of Genoa, Genoa, Italy and SPIN-CNR, Genoa, Italy. Dr Kacmarek is affiliated with the Department of Anesthesiology and Critical Care and the Department of Respiratory Care, Massachusetts General Hospital, Boston, Massachusetts.
}

patients to relieve dyspnea and bronchoconstriction. ${ }^{1} \mathrm{Re}-$ cent studies report a marked variability in nebulization techniques and devices used. In addition, the fraction of the drug dose actually delivered has been shown to depend on many factors, including nebulizing device, ${ }^{2}$ nebulizer position in the delivery circuit, ${ }^{3,4}$ minute ventilation, and breathing pattern. ${ }^{2,4-6}$ During spontaneous breathing in pa-

\footnotetext{
Dr Kacmarek has disclosed relationships with Covidien and Venner Medical. The other authors have disclosed no conflicts of interest.

Correspondence: Lorenzo Ball MD, IRCCS AOU, San Martino-IST, Department of Surgical Sciences and Integrated Diagnostics, University of Genoa, Largo Rosanna Benzi 8, 16132 Genova, Italy. E-mail: lorenzo.ball@edu.unige.it.
}

DOI: $10.4187 /$ respcare. 04275 
tients with tracheostomy, different combinations of corrugated tubing and capping have been studied to act as a reservoir for aerosol droplets, to maximize albuterol delivery in children ${ }^{2}$ and adults. ${ }^{5}$

\section{See the Related Editorial on Page 389}

Pressure support ventilation and CPAP are being used increasingly in critically ill patients. ${ }^{7}$ High-flow CPAP provides relief of upper airway obstruction as well as a reduction of the work of breathing imposed by intrinsic PEEP. ${ }^{8-11}$ High flow is necessary to maintain a constant airway pressure throughout the ventilatory cycle; guidelines suggest that a minimum flow of $60 \mathrm{~L} / \mathrm{min}$ is adequate for most patients. ${ }^{12}$ Nonetheless, in clinical conditions where a high minute ventilation and/or a high peak inspiratory flow are achieved, even higher flows may be necessary to ensure airway stability, especially during the inspiratory phase. ${ }^{8,11}$ Chiumello et al ${ }^{11}$ suggest that the CPAP flow should be maintained at least equal to the patient's peak inspiratory flow, or between 2 and 4 times the patient's minute volume.

High-flow CPAP can be generated by different methods: through mechanical ventilators, with gas blenders, and using oxygen-driven Venturi systems. The last system is composed of a single-limb breathing circuit: it is frequently used during weaning and in acute respiratory failure because of its low cost, ease of transportation, and simple operation. These systems require only a single conventional oxygen supply, ${ }^{8,11,13}$ making them suitable for use also outside the intensive care unit. ${ }^{11,13}$ There are, however, limited data reporting aerosol delivery efficacy when CPAP is used with these devices. ${ }^{14}$ High-flow CPAP circuits can be connected to the patient through artificial airways (endotracheal tubes or tracheostomy cannulas) and with noninvasive interfaces (masks and helmets).

The aim of this study was to investigate the effect of different delivery circuit configurations, nebulizer positions, CPAP levels, and supplementary gas flow on the amount of aerosol delivered to the artificial airway or to the noninvasive interface gas inlet during simulated spontaneous breathing in an in vitro model of an adult patient. For spontaneously breathing patients, we tested 3 different combinations of corrugated tubing and nebulizer positions, and in CPAP circuits, we tested 3 commonly used pressure levels in 3 conditions of flow: $60 \mathrm{~L} / \mathrm{min}$, as suggested by guidelines in most patients; $90 \mathrm{~L} / \mathrm{min}$ for a patient with high minute ventilation; and $30 \mathrm{~L} / \mathrm{min}$, simulating an underpowered CPAP system, as occurs with Venturi systems when a high $\mathrm{F}_{\mathrm{IO}_{2}}$ is set. ${ }^{8}$ We hypothesized that aerosol bronchodilator delivery is affected by the position of the nebulizer, the configuration of the delivery circuit, the CPAP level, and the gas flow.

\section{QUICK LOOK}

\section{Current knowledge}

Nebulized bronchodilators are frequently administered to mechanically ventilated and spontaneously breathing patients. The amount of drug effectively delivered to the patient is affected by several factors, such as ventilation mode, minute ventilation, and type of nebulizer.

\section{What this paper contributes to our knowledge}

In this in vitro model simulating a spontaneously breathing patient, nebulized albuterol delivery was affected by the position of the nebulizer in the respiratory circuit, the level of CPAP, and the gas flow. Without CPAP, a T-piece with a cap at one extremity maximized albuterol delivery. During CPAP, the highest drug delivery rates were achieved with the nebulizer placed proximal to the patient at higher pressures.

\section{Methods}

As illustrated in Figure 1, a pneumatic lung simulator (Dimar, Mirandola, Italy) was connected to different circuits for aerosol delivery (Fig. 2) through a 15-cm catheter mount (Covidien, Dublin, Ireland). Between the lung simulator and the catheter mount, a collection filter was interposed to absorb the delivered albuterol aerosol.

\section{Lung Simulator and Nebulizer Testing Conditions}

The pneumatic lung simulator generated a sinusoidal wave and was set to mimic a mildly tachypneic breathing pattern of an adult: $500 \mathrm{~mL}$ tidal volume, $30 \mathrm{~L} / \mathrm{min}$ peak inspiratory flow rate, and $20 / \mathrm{min}$ breathing frequency. A Hudson Micro Mist nebulizer (Teleflex Hudson RCI, Morrisville, North Carolina) was used to generate the aerosol. The nebulizer was filled with a total volume of $3 \mathrm{~mL}$ of $0.9 \%$ saline solution containing $5 \mathrm{mg}$ of albuterol and operated for $12 \mathrm{~min}$.

\section{Experimental Settings}

Figure 2 illustrates the different circuits used to deliver albuterol. Five different configurations were tested: configuration $\mathrm{A}$, nebulizer distal to the catheter mount and no CPAP; configuration $\mathrm{B}$, nebulizer proximal to the catheter mount and no CPAP; configuration $\mathrm{C}$, nebulizer connected to the catheter mount through a capped T-piece; configuration $\mathrm{D}$, nebulizer placed distal to the catheter mount on the gas supply tube and CPAP delivered through a highflow generator; and configuration E, nebulizer placed prox- 


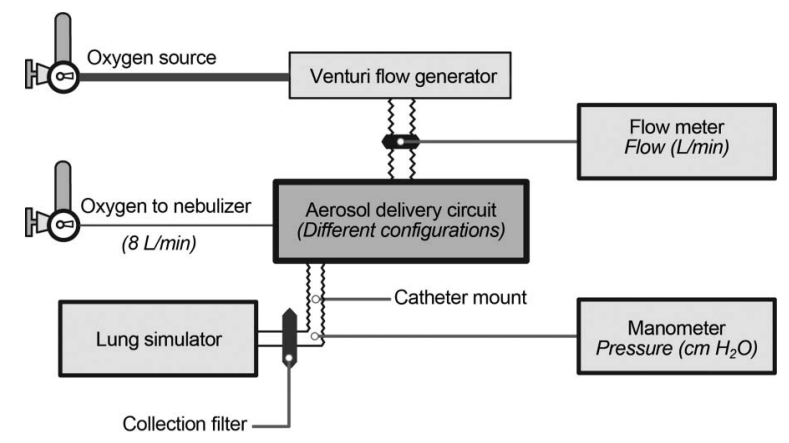

Fig. 1. Experimental setting. The collection filter was placed at a higher level than the rest of the delivery circuit to avoid gravitational dripping of aerosol from the circuit into the filter.

imal to the catheter mount and CPAP delivered through a high-flow generator. In configurations $\mathrm{A}, \mathrm{B}$, and $\mathrm{C}$ the only gas flow was that provided by the flow driving the nebulizer $(8 \mathrm{~L} / \mathrm{min})$.

Two independent oxygen sources were used to supply the circuits: the first one was connected to the nebulizer at a fixed flow rate of $8 \mathrm{~L} / \mathrm{min}$, set with a flow meter (Floval SE, Air Liquide Medical Systems, Paris, France) according to the nebulizer's manufacturer recommendations; the second was connected to a high-flow Venturi generator (Whisper Flow, Philips Respironics, Amsterdam, Netherlands) to provide CPAP for configurations $\mathrm{D}$ and $\mathrm{E}$. The output flow of the Whisper Flow system was measured with a pneumotachograph (ICU-Lab, KleisTEK, Bari, Italy) placed between the flow generator outlet and the delivery circuit (Fig. 2).

Configurations $\mathrm{D}$ and $\mathrm{E}$ were tested with 3 different flows (30, 60, and $90 \mathrm{~L} / \mathrm{min})$ and $3 \mathrm{CPAP}$ levels $\left(5 \mathrm{~cm} \mathrm{H}_{2} \mathrm{O}\right.$, $10 \mathrm{~cm} \mathrm{H}_{2} \mathrm{O}$, and $15 \mathrm{~cm} \mathrm{H}_{2} \mathrm{O}$ ). All flow-pressure combinations were tested. CPAP level was set with an adjustable PEEP valve (KM-809, Koo Medical Equipment, Shanghai, China) and measured at a lung simulator end-expiratory pause with a probe inserted into the catheter mount connected to a digital manometer (ICU-Lab, KleisTEK, Bari, Italy). The PEEP valve acted also as the exhalation port.

\section{Collection Filter and Albuterol Detection}

A custom collection filter was built inserting $7.5 \times 4.55 \mathrm{~cm}$ of general purpose $90-\mathrm{g} / \mathrm{m}^{2}$ absorbent paper into a commercial ventilator circuit filter (DAR, Covidien, Dublin, Ireland). The absorbent paper was preferred to the original hydrophobic membrane of the filter because of its higher capacity to trap aerosol droplets.

After each test, the custom filter was opened, and the absorbent paper was removed and washed with $10 \mathrm{~mL}$ of $0.9 \%$ saline solution and then shaken for 1 min with a vortex oscillator (ZX3, VELP Scientifica, Monza, Italy).
One $\mathrm{mL}$ of the solution was transferred to a quartz cuvette and absorbance measured with a spectrophotometer (Lambda 35, PerkinElmer, Waltham, Massachusetts), operating at a wavelength of $276 \mathrm{~nm}$. This technique was adapted from the current literature. ${ }^{2,4,5}$

\section{Statistics}

Each configuration was evaluated by averaging the results from 3 experimental trials, at each flow and CPAP level. Delivery efficiency was expressed as the percentage of the initial dose that reached the collection filter in each measurement, calculated with the following formula: ( $\mu \mathrm{g}$ of albuterol detected $/ 5,000 \mu \mathrm{g}) \times 100$. The role of configuration, flow, and CPAP level was assessed by univariate analysis of variance, with Bonferroni correction for post hoc multiple comparisons. All statistical analysis was performed with SPSS 21 (IBM, Chicago, Illinois). Data are reported as mean $\pm \mathrm{SD}$, if not otherwise specified. All tests were 2-tailed, and statistical significance was considered as $P<.05$.

\section{Results}

Without CPAP, albuterol delivery was higher with configuration $\mathrm{C}(9.9 \pm 1.1 \%)$, compared with $\mathrm{A}(3.9 \pm 0.1 \%)$ and $\mathrm{B}(5.3 \pm 0.2 \%)$, as shown in Figure $3(P<.001$ in both cases).

With CPAP, albuterol delivery was affected by circuit configuration $(P<.001)$, levels of CPAP $(P<.001)$, and different supplementary flows $(P<.001)$. Albuterol delivery was higher for configuration $\mathrm{E}$ $(13.8 \pm 4.4 \%)$ than $\mathrm{D}(4.5 \pm 3.0 \%)$ at any CPAP level and flow setting $(P<.001$ for all pairwise comparisons; see Fig. 4). In configuration E, albuterol delivery was not affected by an increase of flow, with no significant difference among tested flows. On the other hand, in configuration D, albuterol delivery was lower at $90 \mathrm{~L} /$ min compared with $30 \mathrm{~L} / \mathrm{min}(P=.02)$. The effects of flow, CPAP level, and configuration on albuterol delivery are illustrated in Figure 4.

Overall, albuterol delivery was higher in configurations with CPAP compared with others, with average delivery at any CPAP and flow settings being $9.1 \pm 6.0$ and $6.2 \pm 2.8 \%$, respectively $(P=.03)$. CPAP at $15 \mathrm{~cm} \mathrm{H}_{2} \mathrm{O}$ resulted in the highest albuterol delivery $(P=.02)$ among CPAP configurations.

\section{Discussion}

In this experimental model, we found that: (1) without CPAP, the highest albuterol delivery was obtained capping one outlet of the T-piece (configuration C); (2) during CPAP, the greatest delivery was achieved plac- 

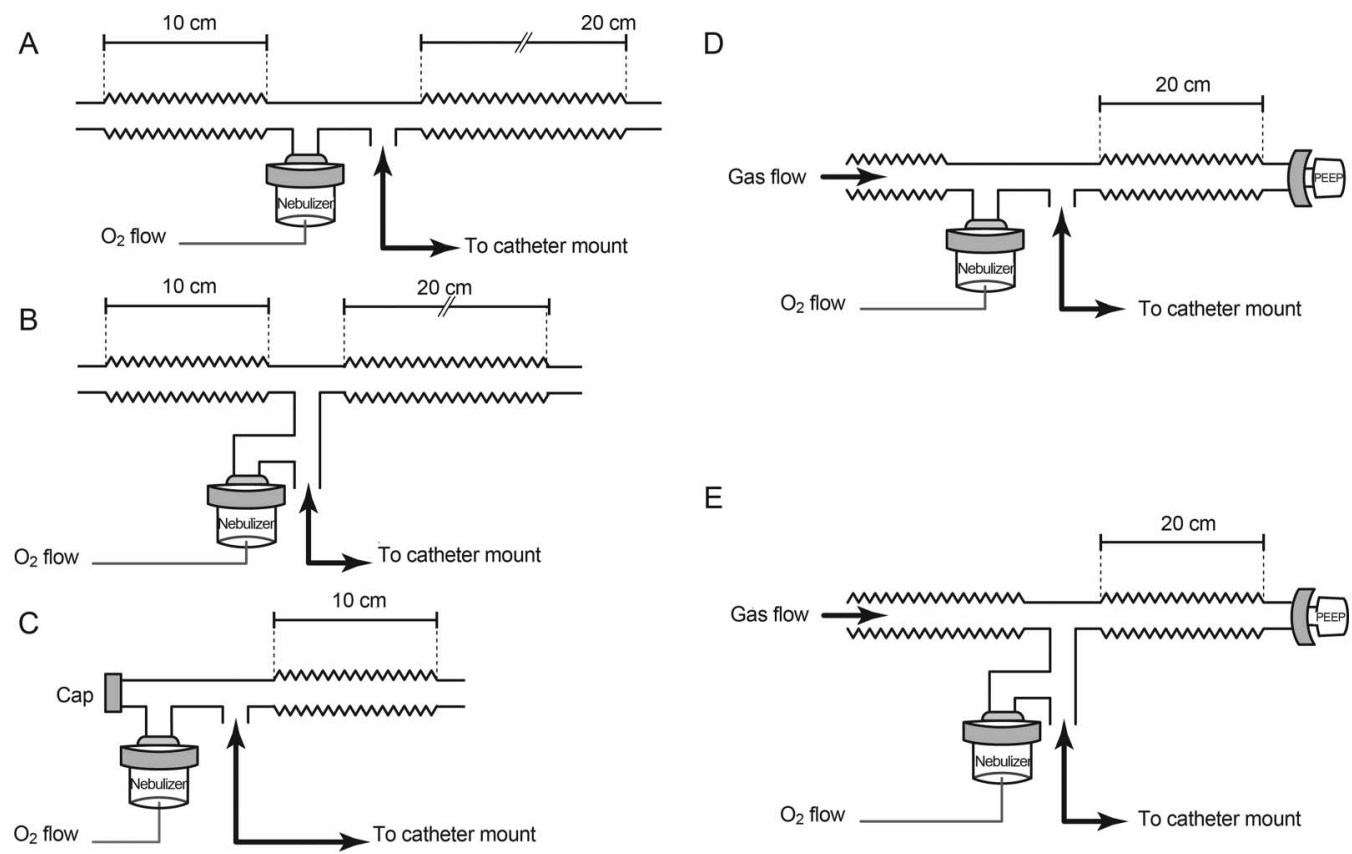

Fig. 2. Different tested configurations. The left panel shows configurations without CPAP (A, B, and C). The right panel shows positive pressure configurations with the nebulizer placed distal (D) or proximal $(E)$ to the collection filter. The catheter mount was connected to the lung simulator as shown in Figure 1.

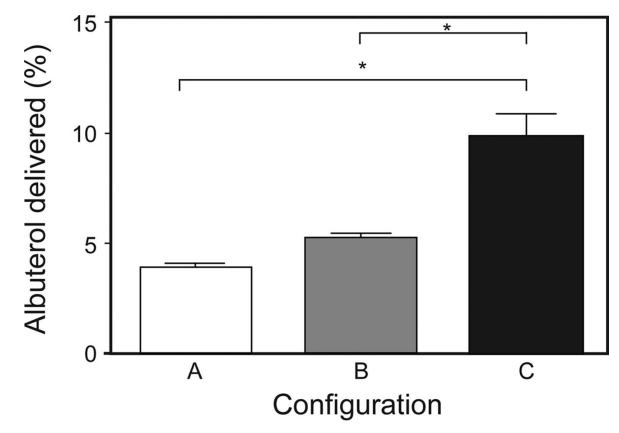

Fig. 3. Albuterol delivery in configurations without CPAP. A: nebulizer distal to the catheter mount and no CPAP; B: nebulizer proximal to the catheter mount and no CPAP; C: nebulizer connected to the catheter mount through a capped T-piece. ${ }^{*}=P<.001$.

ing the nebulizer proximal to the catheter mount after the T-piece connecting the gas supply tubing to the PEEP valve (configuration E); (3) aerosol delivery during CPAP increased as CPAP level increased; and (4) with configuration $\mathrm{E}$, aerosol delivery was independent of flow.

In in-vitro spontaneous breathing models, the amount of aerosol delivered without CPAP has been reported as ranging from 1 to $20 \% .^{5}$ In patients receiving conventional mechanical ventilation, aerosol delivery depends on ventilation mode, tidal volume, breathing frequency, inspiratory flow humidification, nebulizer position, and type of nebulization device, and the delivery effectiveness has been reported around 10\%.15-17 In our study, the amount of albuterol delivered to the lung simulator varied from 0.6 to $18.4 \%$. These data suggest that, independent of the technique used, bronchodilator delivery is a low fraction of the full dose available in the nebulizer.

Without CPAP, the highest albuterol delivery was obtained with configuration $\mathrm{C}$ when a cap was positioned at one outlet of the T-piece and the nebulizer was placed between the capped outlet and the patient (Fig. 2). In configuration $\mathrm{C}$, during expiration, part of the drug was washed out from the corrugated tubing opened to room air, whereas some drug was concentrated in the capped T-piece, which acted as a reservoir. In configurations A and B, the drug in the delivery circuit is diluted in the corrugated tubing and washed-out during expiration, and the reservoir effect was less favorable. Among these 2 low-performing configurations, the nebulizer positioning had no significant effect (Fig. 3). The capped T-piece acts as a true reservoir, since the drug is accumulated during expiration with minimal loss of aerosol to the room. In addition, the presence of 2 segments of corrugated tubing in configurations A and B results in a higher dead space compared with configuration $\mathrm{C}$, potentially hampering $\mathrm{CO}_{2}$ removal in vivo. Our results are in line with those previously reported by Piccuito et al, ${ }^{5}$ where the highest delivery was achieved with a configuration similar to our configuration $\mathrm{C}$, with an additional tubing connected between the nebulizer and the T-piece.

To our knowledge, none of the previous in vitro studies investigated the effects of different flows and CPAP levels 

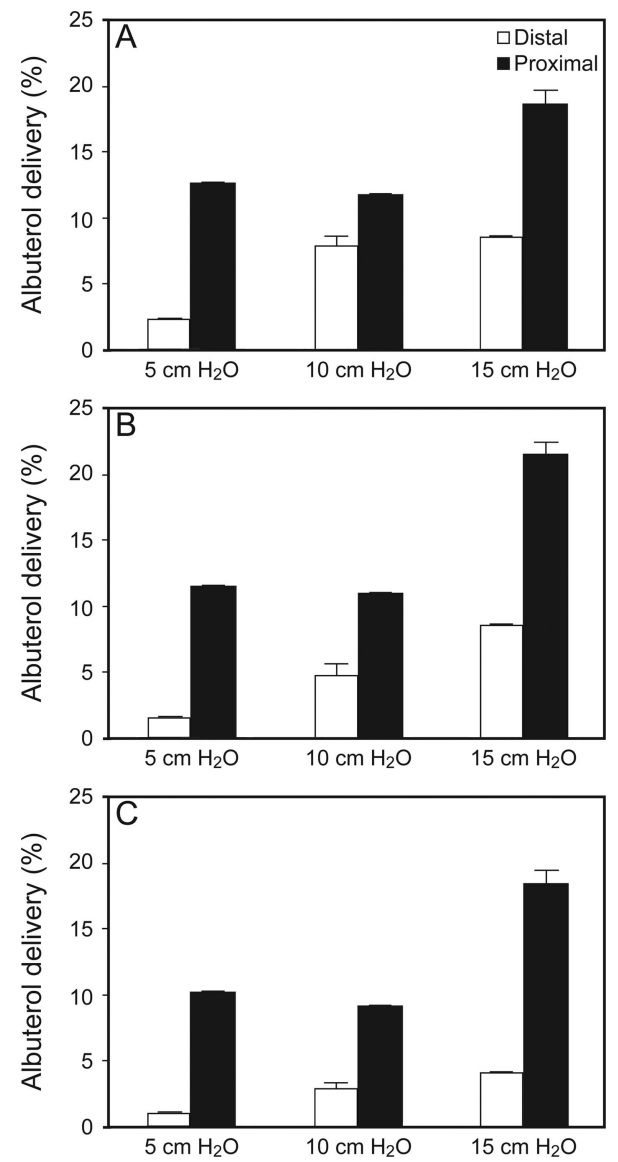

Fig. 4. Efficiency of aerosol bronchodilator delivery during continuous high-flow system at different CPAP and flow levels. A: 30 L/min; B: $60 \mathrm{~L} / \mathrm{min}$; C: $90 \mathrm{~L} / \mathrm{min}$. In all CPAP/flow combinations, the configuration with the nebulizer placed proximal to the catheter mount with CPAP delivered through a high-flow generator delivered higher amounts of salbutamol than the configuration with the nebulizer placed distal to the catheter mount with CPAP delivered through a high-flow generator. $P<.001$ in all cases.

on aerosol delivery. In spontaneous breathing, the delivery circuit can be viewed as composed of 2 parts: (1) a lowflow segment from the patient to the T-piece, whose flow is the patient's minute ventilation and (2) a high-flow segment from the flow source to the PEEP valve, whose flow is that supplied by the flow generator. When the nebulizer is placed in the first segment (configuration E), the drug is washed out through the PEEP valve only during expiration, whereas during the rest of the ventilatory cycle, the aerosol droplets can accumulate within the circuit. When the nebulizer is placed in the second segment (configuration D), a greater washout throughout the whole ventilatory cycle occurs due to the continuous high flow from the flow generator to the adjustable PEEP valve. The hypothesis that high flow is the main cause of aerosol loss is supported by the fact that changing nebulizer position in the absence of supplementary flow and CPAP (configura- tions $\mathrm{A}$ and B) did not lead to major changes in drug delivery (see Fig. 3).

Aerosol delivery was not affected by changes in flow from 30 to $90 \mathrm{~L} / \mathrm{min}$, except when the nebulizer was placed in the low flow limb (configuration E). This suggests that correct nebulizer positioning may allow increased flows, as recommended to optimize CPAP in patients with high minute ventilation. ${ }^{11,18}$ Previous studies suggest that aerosol delivery was improved with a low inspiratory flow. 3,6,19 In our model, the patient breathing pattern was constant, as was inspiratory flow. These results suggest that placing the nebulizer near the catheter mount attenuates the effect of supplemental high flow.

Increasing CPAP from 5 to $15 \mathrm{~cm} \mathrm{H}_{2} \mathrm{O}$ progressively increased aerosol delivery with configuration $\mathrm{D}$, whereas it only increased aerosol delivery at $15 \mathrm{~cm} \mathrm{H}_{2} \mathrm{O}$ with configuration $\mathrm{E}$. The mechanism involved in a higher aerosol delivery at higher CPAP levels is unclear. We hypothesize that this effect is mediated by alterations in the gas dynamics within the delivery circuit, possibly due to a modification in aerosol droplet size and motion or to higher gas turbulence, resulting in prolonged aerosol accumulation within the tubing, decreasing drug wash-out.

Our study has some limitations. First, we used an in vitro model in which aerosol delivery was only measured at the lung simulator inlet. Our data may overestimate the dose actually delivered to the airways; however, it has been estimated that albuterol trapped by the tracheostomy tube was below $1 \%$ of the delivered dose. ${ }^{2}$ Second, the possible effects of different interfaces between the patient and the circuit as well as the affect of different modes of mechanical ventilation were not evaluated. Third, the effects of humidity and breathing pattern were not examined.

\section{Conclusions}

We conclude that, without CPAP, a T-piece with a cap at one outlet maximizes albuterol delivery. During CPAP, the nebulizer should always be placed as close to the patient as possible, and the highest CPAP levels clinically indicated should be set. These results have a straightforward application in clinical practice when these CPAP circuits are connected to an artificial airway, whereas the role of different noninvasive interfaces, such as masks or helmets, should be investigated in further studies.

\section{REFERENCES}

1. Manthous CA, Hall JB. Administration of therapeutic aerosols to mechanically ventilated patients. Chest 1994;106(2):560-571.

2. Berlinski A. Nebulized albuterol delivery in a model of spontaneously breathing children with tracheostomy. Respir Care 2013;58(12): 2076-2086.

3. MacIntyre NR. Aerosol delivery through an artificial airway. Respir Care 2002;47(11):1279-1288, discussion 1285-1279. 
4. Dai B, Kang J, Sun LF, Tan W, Zhao HW. Influence of exhalation valve and nebulizer position on albuterol delivery during noninvasive positive pressure ventilation. J Aerosol Med Pulm Drug Deliv 2014;27(2):125-132.

5. Piccuito CM, Hess DR. Albuterol delivery via tracheostomy tube. Respir Care 2005;50(8):1071-1076.

6. Dolovich MA. Influence of inspiratory flow rate, particle size, and airway caliber on aerosolized drug delivery to the lung. Respir Care 2000;45(6):597-608.

7. Esteban A, Frutos-Vivar F, Muriel A, Ferguson ND, Peñuelas O, Abraira V, et al. Evolution of mortality over time in patients receiving mechanical ventilation. Am J Respir Crit Care Med 2013;188(2): 220-230.

8. Glover GW, Fletcher SJ. Assessing the performance of the Whisperflow continuous positive airway pressure generator: a bench study. Br J Anaesth 2009;102(6):875-881.

9. Caruso P, Fu C, Ribeiro de Carvalho CR, Fletcher SJ. Continuous flow positive airway pressure generator in critically ill patients. Br J Anaesth 2010;104(1):116, author reply 116-117.

10. Fu C, Caruso P, Lucatto JJ, de Paula Schettino GP, de Souza R, Carvalho CR. Comparison of two flow generators with a noninvasive ventilator to deliver continuous positive airway pressure: a test lung study. Intensive Care Med 2005;31(11):1587-1591.

11. Chiumello D, Esquinas AM, Moerer O, Terzi N. A systematic technical review of the systems for the continuous positive airway pressure. Minerva Anestesiol 2012;78(12):1385-1393.

12. British Thoracic Society Standards of Care Committee. Non-invasive ventilation in acute respiratory failure. Thorax 2002;57(3):192211.
13. Isgrò S, Zanella A, Giani M, Abd El Aziz El Sayed Deab S, Pesenti A, Patroniti N. Performance of different PEEP valves and helmet outlets at increasing gas flow rates: a bench top study. Minerva Anestesiol 2012;78(10):1095-1100.

14. Hess DR. The mask for noninvasive ventilation: principles of design and effects on aerosol delivery. J Aerosol Med 20(Suppl 1)07;20: S85-S98, discussion S98-S99.

15. Hess DR, Dillman C, Kacmarek RM. In vitro evaluation of aerosol bronchodilator delivery during mechanical ventilation: pressure-control vs volume control ventilation. Intensive Care Med 2003;29(7): 1145-1150.

16. Thomas SH, Harvey C, Page CJ, Treacher DF, O’Doherty MJ. Aerosol deposition in mechanically ventilated patients. Am J Respir Crit Care Med 1994;150(5 Pt 1):1474-1475.

17. Wan GH, Lin HL, Fink JB, Chen YH, Wang WJ, Chiu YC, et al. In vitro evaluation of aerosol delivery by different nebulization modes in pediatric and adult mechanical ventilators. Respir Care 2014; 59(10):1494-1500.

18. Organized jointly by the American Thoracic Society, the European Respiratory Society, the European Society of Intensive Care Medicine, and the Société de Réanimation de Langue Française, and approved by ATS Board of Directors, December 2000. International Consensus Conferences in Intensive Care Medicine: noninvasive positive pressure ventilation in acute respiratory failure. Am J Respir Crit Care Med 2001;163(1):283-291.

19. Dhand R. Maximizing aerosol delivery during mechanical ventilation: go with the flow and go slow. Intensive Care Med 2003;29(7):1041-1042.

This article is approved for Continuing Respiratory Care Education credit. For information and to obtain your CRCE

(free to AARC members) visit

www.rcjournal.com

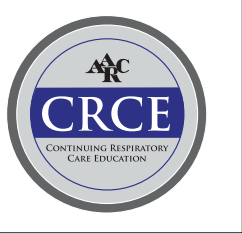

